\title{
REDUCED ORDER COMPUTATIONAL CONTINUA
}

\author{
Jacob Fish \\ Columbia University \\ New York, NY 10024, USA \\ fishj@columbia.edu
}

\author{
Vasilina Filonova \\ Columbia University \\ New York, NY 10024, USA \\ vf2197@columbia.edu
}

\author{
Zheng Yuan \\ Multiscale Design Systems \\ New York, NY 10010, USA \\ cto@multiscale.biz
}

\section{INTRODUCTION}

The paper presents a new multiscale framework that is both mathematically rigorous and practical in the sense that it has been successfully applied in aerospace, automotive and civil engineering industries. The "rigor" aspect of the method is provided by recently developed computational continua $\left(\mathrm{C}^{2}\right)$ formulation (Fish and Kuznetsov, 2009), which is endowed with fine-scale details, introduces no scale separation, makes no assumption about infinitesimality of the fine-scale structure, does not require higher order continuity, introduces no new degrees-of-freedom and is free of higher order boundary conditions. The "practicality" aspect of the proposed method is inherited from the reduced order homogenization (Yuan and Fish, 2009, Fish and Yuan, 2008) approach, which constructs residual free-fields that eliminate the bottleneck of satisfying fine-scale equilibrium equations and is endowed with a hierarchical model improvement capability where the cost of the most inexpensive member of the sequence is comparable to that of semianalytical or phenomenological methods.

Blending of the two methods into a single cohesive computational framework, hereafter to be referred to as the Reduced order Computational Continua or simply $\mathrm{RC}^{2}$, that inherits the underlying characteristics of its two ingredients, is the main objective of the present manuscript. We conclude the manuscript with a brief summary and discussion of future research directions.

In the present manuscript we consider a heterogeneous body formed by a repetition of a fine structure (unit cells) occupying an open, bounded domain $\Omega^{\xi} \subset \square^{3}$. The unit cell domain denoted as $\Theta \subset \square^{3}$ is assumed to be finite, i.e. unlike in the homogenization theories it is not infinitesimally small compared to the coarse-scale domain.

The following governing equations on $\boldsymbol{x} \in \Omega^{\xi}$ are stated at the fine-scale of interest

$$
\begin{aligned}
& \sigma_{i j, j}^{\zeta}(\boldsymbol{x})+b_{i}^{\zeta}(\boldsymbol{x})=0 \text { on } \Omega^{\xi} \\
& \sigma_{i j}^{\zeta}(\boldsymbol{x})=L_{i j k l}^{\zeta}(\boldsymbol{x})\left(\varepsilon_{k l}^{\zeta}(\boldsymbol{x})-\mu_{k l}^{\zeta}(\boldsymbol{x})\right) \text { on } \Omega^{\zeta} \\
& \varepsilon_{i j}^{\zeta}(\boldsymbol{x})=u_{(i, j)}^{\zeta}(\boldsymbol{x}) \equiv \frac{1}{2}\left(u_{i, j}^{\zeta}+u_{j, i}^{\zeta}\right) \text { on } \Omega^{\zeta} \\
& u_{i}^{\zeta}(\boldsymbol{x})=\bar{u}_{i}^{\zeta}(\boldsymbol{x}) \quad \text { on } \partial \boldsymbol{\Omega}^{u \zeta} \\
& \sigma_{i j}^{\zeta}(\boldsymbol{x}) n_{j}^{\zeta}(\boldsymbol{x})=\bar{t}_{i}^{\zeta}(\boldsymbol{x}) \text { on } \partial \boldsymbol{\Omega}^{t \zeta}
\end{aligned}
$$

where $u_{i}^{\zeta}$ denotes displacements; $\sigma_{i j}^{\zeta}$ - the Cauchy stress; $\varepsilon_{i j}^{\zeta}$ - the total small strain; $\mu_{i j}^{\zeta}$ - the eigenstrain arising from inelastic deformation, thermal changes, moisture effects or phase transformation; $b_{i}^{\zeta}-$ the body force, $L_{i j k l}^{\xi}$ - the elastic material properties (Hook's law). The superscript $\zeta$ denotes existence of fine-scale features. $\partial \Omega^{u \zeta}$ and $\partial \Omega^{t \xi}$ denote essential and natural boundary such that $\partial \Omega^{\xi}=\partial \Omega^{u \xi} \cup \partial \Omega^{t \xi}$ and $\partial \Omega^{u \zeta} \cap \partial \Omega^{t \zeta}=\varnothing$.

For simplicity we assume that the body force $b_{i}^{\zeta}(\boldsymbol{x})$ is a smooth function, i.e. it may vary linearly over the unit cell domain.

\section{COMPUTATIONAL CONTINUA}

Following (Fish and Kuznetsov, 2009) we define computational continua domain $\Omega^{c}$ as a disjoint union $\left.{ }^{\mathrm{i}}\right]$ (sometimes called direct sum or free union) of Computational Unit Cell (CUC) domains $\Theta_{\hat{\boldsymbol{x}}_{I}}$

$$
\Omega^{c}=\coprod_{I=1}^{\breve{N}} \Theta_{\hat{\boldsymbol{x}}_{I}}
$$

where $\hat{\boldsymbol{x}}_{I}$ denotes the coordinates of centroid of the CUC domain $\Theta_{\hat{x}_{I}}$ and $\hat{N}$ the number of computational unit cells. Note that if $\Theta_{\hat{x}_{I}} \cap \Theta_{\hat{x}_{J}}=0, \forall I \neq J$ then the disjoint union reduces to a regular union. The position of the CUC centroid, $\hat{\boldsymbol{x}}_{I}$, and quadrature weights are calculated so that the integral over the composite domain $\Omega^{\xi}$ would coincide with the integral over the computational continua domain $\Omega^{c}$. This is accomplished via so-called nonlocal quadrature defined as

$$
\begin{aligned}
& \int_{\Omega^{\xi}} f^{\zeta}(\boldsymbol{x}) d \Omega=\sum_{I=1}^{\hat{N}} \int_{\Theta_{\hat{x}_{I}}} \hat{\omega}\left(\hat{\boldsymbol{x}}_{I}, \chi\right) f^{\zeta}\left(\hat{\boldsymbol{x}}_{I}, \chi\right) d \Theta ; \\
& \hat{\omega}\left(\hat{\boldsymbol{x}}_{I}, \chi\right)=\frac{1}{\left|\Theta_{\hat{\boldsymbol{x}}_{I}}\right|} J^{e}\left(\hat{\boldsymbol{x}}_{I}, \chi\right) \hat{W}_{I}
\end{aligned}
$$


where $J^{e}\left(\hat{\boldsymbol{x}}_{I}, \chi\right)$ is the Jacobean that maps a coarsescale element into a bi-unit cube; $\hat{W}_{I}$ denotes the nonlocal quadrature weight. The nonlocal quadrature leads to the dependence of the position of the unit cell centroid $\hat{\boldsymbol{x}}_{I}$ on the computational unit cell size relative to the coarse-scale finite element size.

For each CUC domain, $\Theta_{\hat{x}_{I}}$, we introduce a local coordinate system with respect to the unit cell centroid defined as $\boldsymbol{\chi}=\boldsymbol{x}-\hat{\boldsymbol{x}}_{I}$. Any function $f^{\zeta}(\boldsymbol{x})$ can be expressed in terms of the local coordinates, $\chi$, and the corresponding CUC centroid, $\hat{\boldsymbol{x}}_{I}$, i.e. $f^{\zeta}(\boldsymbol{x})=f^{\zeta}\left(\hat{\boldsymbol{x}}_{I}, \boldsymbol{\chi}\right)$. Note that unlike in the classical homogenization theory there is no scale separation, i.e. $\boldsymbol{\chi}$ and $\boldsymbol{x}$ are of the same order.

We further assume an additive decomposition of displacements $\boldsymbol{u}^{\zeta}$ into a smooth coarse-scale function $\boldsymbol{u}^{c}$ and an oscillatory weakly periodic function $\boldsymbol{u}^{(1)}$ normalized as

$$
\int_{\Theta_{\hat{x}_{I}}} \boldsymbol{u}^{(1)}\left(\hat{\boldsymbol{x}}_{I}, \boldsymbol{\chi}\right) d \Theta=\mathbf{0}
$$

The total strain $\boldsymbol{\varepsilon}^{\zeta}\left(\hat{\boldsymbol{x}}_{I}, \boldsymbol{\chi}\right)$ is additively decomposed into coarse-scale strain $\boldsymbol{\varepsilon}^{c}$ and fine-scale perturbation $\boldsymbol{\varepsilon}^{*}$ as

$$
\varepsilon_{i j}^{\zeta}\left(\hat{\boldsymbol{x}}_{I}, \boldsymbol{\chi}\right)=\varepsilon_{i j}^{c}\left(\hat{\boldsymbol{x}}_{I}, \boldsymbol{\chi}\right)+\varepsilon_{i j}^{*}\left(\hat{\boldsymbol{x}}_{I}, \chi\right)
$$

defined as symmetric spatial derivative of displacements

$$
\begin{aligned}
\varepsilon_{i j}^{c}\left(\hat{\boldsymbol{x}}_{I}, \boldsymbol{\chi}\right) & =u_{(i, j)}^{c}\left(\hat{\boldsymbol{x}}_{I}, \boldsymbol{\chi}\right) \\
\varepsilon_{i j}^{*}\left(\hat{\boldsymbol{x}}_{I}, \boldsymbol{\chi}\right) & =u_{(i, j)}^{(1)}\left(\hat{\boldsymbol{x}}_{I}, \boldsymbol{\chi}\right)
\end{aligned}
$$

The coarse-scale strain field $\boldsymbol{\varepsilon}^{c}$ is approximated by a linear function over the computational unit cell domain consisting of an average strain $\bar{\varepsilon}_{i j}$, and an average strain gradient denoted by $\bar{\varepsilon}_{i j, m}$ defined as

$$
\begin{aligned}
& \varepsilon_{i j}^{c}\left(\hat{\boldsymbol{x}}_{I}, \boldsymbol{\chi}\right)=\bar{\varepsilon}_{i j}\left(\hat{\boldsymbol{x}}_{I}\right)+\bar{\varepsilon}_{i j, m}\left(\hat{\boldsymbol{x}}_{I}\right) \chi_{m} \\
& \bar{\varepsilon}_{i j}\left(\hat{\boldsymbol{x}}_{I}\right)=\frac{1}{\left|\Theta_{\hat{\boldsymbol{x}}_{I}}\right|} \int_{\Theta_{\hat{x}_{I}}} \varepsilon_{i j}^{\zeta}\left(\hat{\boldsymbol{x}}_{I}, \boldsymbol{\chi}\right) d \Theta \\
& \bar{\varepsilon}_{i j, m}\left(\hat{\boldsymbol{x}}_{I}\right)=\frac{1}{\left|\Theta_{\hat{\boldsymbol{x}}_{I}}\right|} \int_{\Theta_{\hat{x}_{I}}} \frac{\partial \varepsilon_{i j}^{\zeta}}{\partial \chi_{m}}\left(\hat{\boldsymbol{x}}_{I}, \boldsymbol{\chi}\right) d \Theta
\end{aligned}
$$

Employing Eq. (8) and constitutive relation in (1) , the equilibrium equation can be expressed as

$$
\begin{aligned}
& \left\{L_{i j k l}(\boldsymbol{\chi})\left[\begin{array}{c}
\bar{\varepsilon}_{k l}\left(\hat{\boldsymbol{x}}_{I}\right)+\bar{\varepsilon}_{k l, m}\left(\hat{\boldsymbol{x}}_{I}\right) \boldsymbol{\chi}_{m}+ \\
u_{(k, l)}^{(1)}\left(\hat{\boldsymbol{x}}_{I}, \boldsymbol{\chi}\right)-\mu_{k l}^{\zeta}\left(\hat{\boldsymbol{x}}_{I}, \boldsymbol{\chi}\right)
\end{array}\right]\right\}_{, j} \\
& +b_{i}^{\zeta}\left(\hat{\boldsymbol{x}}_{I}, \boldsymbol{\chi}\right)=0
\end{aligned}
$$

where, for simplicity, we assume that elastic properties depend on fine scale coordinates only, $L_{i j k l}^{\zeta}(\boldsymbol{x})=L_{i j k l}(\boldsymbol{\chi})$, i.e. they are same for all computational unit cells, $\Theta_{\hat{\boldsymbol{x}}_{I}}$.

\section{RESIDUAL-FREE UNIT CELL PROBLEM}

The bottleneck of multiscale computations is in finescale computations of complex microstructures. To illustrate the computational complexity involved, consider a coarse- scale problem with $N_{\text {cells }}$ quadrature points, $n$ load increments at the coarse scale, and $I_{\text {coarse }}$ and $I_{\text {fine }}$ average iterations at the two scales, respectively. The total number of linear solves of a fine scale problem is thus $N_{\text {cells }} \cdot n \cdot I_{\text {coarse }} \cdot I_{\text {fine }}$ - a formidable computational complexity for large number of complex unit cells. This bottleneck can be successfully removed my constructing residual-free fields that a priori satisfy fine-scale equilibrium equations.

For large unit cells over which the coarse-scale fields are no longer constant the residual-free expansion has to be modified to include higher order residual-free coarse-scale fields. This is accomplished by introducing the following decomposition of fine-scale displacements

$$
\begin{aligned}
& u_{i}^{(1)}\left(\hat{\boldsymbol{x}}_{I}, \boldsymbol{\chi}\right)=H_{i}^{m n}\left(\hat{\boldsymbol{x}}_{I}, \boldsymbol{\chi}\right) \bar{\varepsilon}_{m n}\left(\hat{\boldsymbol{x}}_{I}\right)+ \\
& H_{i}^{m n p}\left(\hat{\boldsymbol{x}}_{I}, \boldsymbol{\chi}\right) \bar{\varepsilon}_{m n, p}\left(\hat{\boldsymbol{x}}_{I}\right)+ \\
& \int_{\Theta_{\hat{\boldsymbol{x}}_{I}}} \tilde{h}_{i}^{m n}\left(\hat{\boldsymbol{x}}_{I}, \boldsymbol{\chi}, \tilde{\boldsymbol{\chi}}\right) \mu_{m n}^{\zeta}\left(\hat{\boldsymbol{x}}_{I}, \tilde{\boldsymbol{\chi}}\right) d \tilde{\Theta}+ \\
& \int_{S_{\hat{x}_{I}}} \breve{h}_{i}^{\check{m}}\left(\hat{\boldsymbol{x}}_{I}, \boldsymbol{\chi}, \tilde{\boldsymbol{\chi}}\right) \delta_{\breve{m}}^{\zeta}\left(\hat{\boldsymbol{x}}_{I}, \breve{\boldsymbol{\chi}}\right) d \breve{S}
\end{aligned}
$$

where $H_{i}^{m n}, H_{i}^{m n p}, \tilde{h}_{i}^{m n}, \breve{h}_{i}^{\breve{m}}$ are strain, strain gradient, eigenstrain, eigenseparation influence functions, respectively; $\delta_{\breve{m}}^{\zeta}$ the eigenseparation; the subscript $\breve{m}$ indicates components in the local Cartesian coordinate system of the CUC interface, denoted by $S_{\hat{x}_{I}}$.

Assume that all influence functions are $\boldsymbol{\chi}$ periodic over the CUC domain. Then integral expressions (8) and (5) are satisfied.

\section{THE INFLUENCE FUNCTIONS PROBLEM}

The influence functions, $H_{i}^{m n}, H_{i}^{m n p}, \tilde{h}_{i}^{m n}, \breve{h}_{i}^{\breve{m}}$, are constructed to satisfy the weak form for arbitrary $\bar{\varepsilon}_{m n}$, $\bar{\varepsilon}_{m n, p}, \mu_{m n}^{\zeta}$, and $\delta_{\breve{m}}^{\zeta}$. Exploiting smoothness condition of the body force yields the following influence function problems 


$$
\begin{aligned}
& \int_{\Theta_{\dot{x}_{1}}} w_{i}^{(1)}\left(\hat{\boldsymbol{x}}_{I}, \chi\right)\left\{L_{i j k l}(\chi) E_{k l}^{m n}\left(\hat{\boldsymbol{x}}_{I}, \chi\right)\right\}_{, j} d \Theta=0 \\
& \int_{\Theta_{\dot{x}_{I}}} w_{i}^{(1)}\left(\hat{\boldsymbol{x}}_{I}, \chi\right)\left\{L_{i j k l}(\chi) E_{k l}^{m n p}\left(\hat{\boldsymbol{x}}_{I}, \chi\right)\right\}_{, j} d \Theta=0 \\
& \int_{\Theta_{\dot{s}_{l}}} w_{i}^{(1)}\left(\hat{\boldsymbol{x}}_{I}, \boldsymbol{\chi}\right)\left\{L_{i j k l}(\chi)\left(\int_{S_{\bar{x}_{l}}} \breve{h}_{(k, l)}^{\bar{m}}\left(\hat{\boldsymbol{x}}_{I}, \boldsymbol{\chi}, \breve{\boldsymbol{\chi}}\right) \delta_{\breve{m}}^{\zeta}\left(\hat{\boldsymbol{x}}_{I}, \breve{\boldsymbol{\chi}}\right) d \breve{S}\right)\right\}_{, j} d \Theta=0 \\
& \int_{\Theta_{\dot{x}_{j}}} w_{i}^{(1)}\left(\hat{\boldsymbol{x}}_{I}, \boldsymbol{\chi}\right)\left\{L_{i j k l}(\boldsymbol{\chi})\left(\begin{array}{l}
\int_{\Theta_{\boldsymbol{i}_{j}}} \tilde{h}_{(k, l)}^{m n}\left(\hat{\boldsymbol{x}}_{I}, \boldsymbol{\chi}, \tilde{\boldsymbol{\chi}}\right) \mu_{m n}^{\zeta}\left(\hat{\boldsymbol{x}}_{I}, \tilde{\boldsymbol{\chi}}\right) d \tilde{\Theta}- \\
\mu_{k l}^{\zeta}\left(\hat{\boldsymbol{x}}_{I}, \boldsymbol{\chi}\right)
\end{array}\right)\right\}_{, j} d \Theta
\end{aligned}
$$

The above euations define the weak form of the boundary value problems for periodic (or weakly periodic) influence functions, $H_{i}^{m n}, H_{i}^{m n p}, \tilde{h}_{i}^{m n}, \breve{h}_{i}^{\breve{m}}$, respectively.

\section{MODEL REDUCTION}

The primary objective of the model reduction is to reduce the computational complexity of solving a sequence of unit cell problems. This is accomplished by discretizing eigenstrains by $C^{-1}\left(\Theta_{\hat{x}_{I}}\right)$ continuous piecewise linear function

$$
\begin{aligned}
& \mu_{k l}^{\zeta}\left(\hat{\boldsymbol{x}}_{I}, \chi\right)=\sum_{\alpha=1}^{n} \tilde{N}^{(\alpha)}\left(\hat{\boldsymbol{x}}_{I}, \chi^{(\alpha)}\right) \mu_{k l}^{(\alpha)}\left(\hat{\boldsymbol{x}}_{I}\right) \\
& \mu_{i j}^{(\alpha)}\left(\hat{\boldsymbol{x}}_{I}, \boldsymbol{\chi}^{(\alpha)}\right) \equiv \bar{\mu}_{i j}^{(\alpha)}\left(\hat{\boldsymbol{x}}_{I}\right)+\bar{\mu}_{i j, m}^{(\alpha)}\left(\hat{\boldsymbol{x}}_{I}\right) \chi_{m}^{(\alpha)} \\
& \tilde{N}^{(\alpha)}\left(\hat{\boldsymbol{x}}_{I}, \chi^{(\alpha)}\right) \equiv \begin{cases}1 & \chi^{(\alpha)} \in \Theta_{\hat{\boldsymbol{x}}_{I}}^{(\alpha)} \\
0 & \chi^{(\alpha)} \notin \Theta_{\hat{\boldsymbol{x}}_{t}}^{(\alpha)}\end{cases} \\
& \Theta_{\hat{\boldsymbol{x}}_{t}}=\bigcup_{\alpha=1}^{n} \Theta_{\hat{\boldsymbol{x}}_{t}}^{(\alpha)} ; \varnothing=\bigcap_{\alpha=1}^{n} \Theta_{\hat{\boldsymbol{x}}_{t}}^{(\alpha)}
\end{aligned}
$$

and eigenseparations by $C^{0}\left(\Theta_{\hat{\boldsymbol{x}}_{I}}\right)$ piecewise linear function

$$
\begin{aligned}
& \delta_{\dot{q}^{\xi}}\left(\hat{\boldsymbol{x}}_{I}, \chi\right)=\sum_{\xi=1}^{m} \breve{N}^{(\xi)}\left(\hat{\boldsymbol{x}}_{I}, \chi\right) \delta_{\breve{q}}^{(\xi)}\left(\hat{\boldsymbol{x}}_{I}\right) \\
& \breve{N}^{(\xi)}\left(\hat{\boldsymbol{x}}_{I}, \chi\right) \equiv \begin{cases}\sum_{r \in S_{\dot{x}_{I}}^{(\xi)}} N_{r}\left(\hat{\boldsymbol{x}}_{I}, \chi\right) & \chi \in S_{\dot{x}_{I}}^{(\xi)} \\
0 & \chi \notin S_{\dot{x}_{I}}^{(\xi)}\end{cases}
\end{aligned}
$$

where $\bar{\mu}_{i j}^{(\alpha)}\left(\hat{\boldsymbol{x}}_{I}\right)$ and $\bar{\mu}_{i j, m}^{(\alpha)}\left(\hat{\boldsymbol{x}}_{I}\right)$ are the average and linear variation of the eigenstrain, respectively; $\chi^{(\alpha)}=\chi-\hat{\chi}^{(\alpha)}$ denotes the local coordinate system positioned at the centroid $\hat{\chi}^{(\alpha)}$ of phase partition $\alpha$; $\Theta_{\hat{\boldsymbol{x}}_{I}}^{(\alpha)}$ is the volume of partition $\alpha$ in the CUC domain $\Theta_{\hat{x}_{I}}$ and $n$ is a number of such non-overlapping partitions; $S_{\hat{\mathbf{x}}_{I}}^{(\xi)}$ denotes $\xi$ surface partition in $S_{\hat{x}_{I}}$ and $m$ is the total number of surface partitions; $\breve{N}^{(\xi)}$ is chosen to be $C^{0}\left(\Theta_{\hat{\boldsymbol{x}}_{I}}\right)$ to ensure smoothness of interface separations constructed by a sum of finite element shape functions $N_{r}$ defined over the interface partition $S_{\hat{x}_{I}}^{(\xi)}$.

\section{NUMERICAL EXAMPLES}

For model verification we consider a beam consisting of eight coarse-scale elements. For simplicity, both the coarse-scale element and the computational unit cell are in the form of a cube. The unit cell contains a spherical or cylindrical inclusion placed at the CUC centroid as shown in Fig. 1. The diameter of the inclusion crosssection is chosen to be 0.6 of the CUC length. For the reference solution, we consider direct numerical simulation (DNS), which employs sufficiently fine finite element mesh where the element size is considerably smaller than the size of the inclusion. The results of the reduced order computational continua (referred here as $\mathrm{RC}^{2}$ ) are also compared to the $O(1)$ reduced order formulation (subsequently referred as $O(1)$ ).

We study the dependence of the solution accuracy on CUC size. For the $\mathrm{RC}^{2}$ formulation any value of $\zeta$ in $[0,1]$ can be considered. However, the reference solution cannot be constructed for some choices of $\zeta$, such as for instance $1>\zeta>0.5$, if the coarse-scale problem is made by spatial repetition of complete unit cells. Thus the reference solution is constructed for $\zeta=1, \zeta=0.5$, which corresponds to coarse-scale element made of one and eight CUCs, respectively. Note that the results obtained by $O(1)$ homogenization method do not depend on the value of $\zeta$.

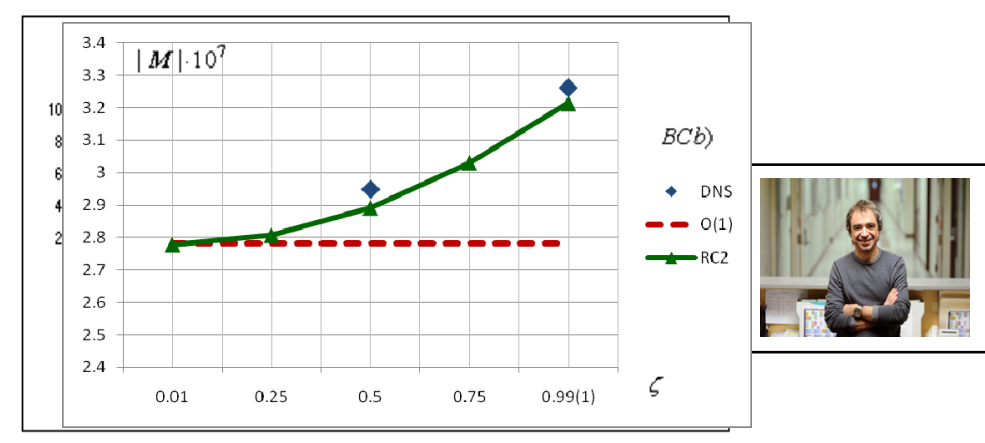

Figures 1: Comparisonof moments for various CUC sizes

It can be seen that $\mathrm{RC}^{2}$ solution agrees well with the DNS. For $\zeta=1$ the $O(1)$ formulation results in $25 \%$ error in moment for boundary conditions of type $a$ ) 
(Fig. 6, top left) and 15\% error for boundary condition of type $b$ ). (Fig. 6, bottom). As in the previous cases the $\mathrm{RC}^{2}$ and $O(1)$ formulations coincide at $\zeta=0.01$.

\section{REFERENCES}

Fish, J. and Kuznetsov, S. "Computational Continua," International Journal for Numerical Methods in Engineering, Vol. 84, Issue 7, pp. 774-802, 2010

Yuan, Z. and Fish, J., "Multiple Scale EigendeformationBased Reduced Order Homogenization," Comp. Meth. Appl. Mech. Engng., 198 (21-26), pp. 2016-2038, 2009.

Fish, J. and Yuan, Z. in J. Fish (ed), "Bridging the Scales in Science and Engineering," Oxford University Press, 2008.

\section{AUTHOR BIOGRAPHY}

JACOB FISH was born in Vilnius, Lithuania, went to Technion in Israel for BS and MS degreesand Northwestern University in US where he obtained his $\mathrm{PhD}$ degree in 1989. Over the past 27 years (in both industry and academia) Dr. Fish, the Carleton Professor of Engineering and Past President f US Association for
Compuational Mechanics, has been in the forefront of multiscale computational science and engineering, an emerging discipline that bridges the gap between modeling, simulation and design of products based on multiscale principles. He has an accomplished track record of technology transfer to industry. His multiscale methodologies have been employed by industry for manufacturing processes of GE90 fan blades; design of turbo-engines for Allison Engines, GE and RollsRoyce; simulation of aerospace structural components for Lockheed-Martin and Sikorski; optimization of energy absorption mechanism for lightweight composite cars manufactured by Ford, GM and Chrysler; predicting environmental degradation of polyimidebased composites in collaboration with Boeing, GE Aviation and Renegade Materials; analysis of concrete targets subjected to impact loading by high speed projectiles; design of piezoelectric and ferroelectric materials; and numerous nanotechnology applications including nanodevices and nanocomposites sponsored by Northrop-Grumman, Sandia National Laboratory, Army Research Laboratory and Department of Energy.

[i] Armstrong, M. A. Basic Topology, rev. ed. New York: Springer-Verlag, (1997). 